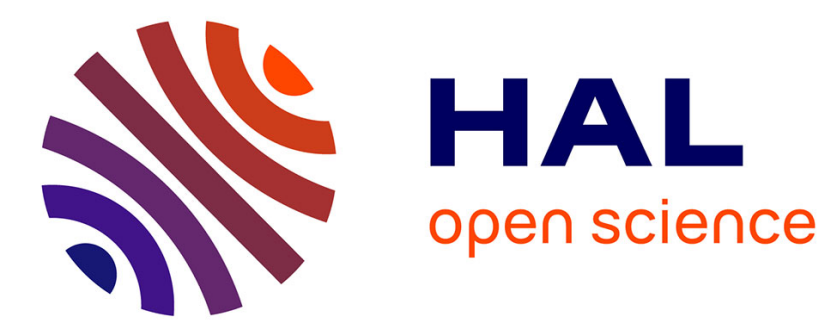

\title{
De la philosophie morale humienne à l'éthique du care : un billet aller-retour
}

Céline Bonicco-Donato

\section{To cite this version:}

Céline Bonicco-Donato. De la philosophie morale humienne à l'éthique du care: un billet aller-retour . Cahiers philosophiques, 2014, Le care: éthique et politique, 136, p. 7-23. 10.3917/caph.136.0007 . hal-01493219

\section{HAL Id: hal-01493219 https://hal.science/hal-01493219}

Submitted on 21 Mar 2017

HAL is a multi-disciplinary open access archive for the deposit and dissemination of scientific research documents, whether they are published or not. The documents may come from teaching and research institutions in France or abroad, or from public or private research centers.
L'archive ouverte pluridisciplinaire HAL, est destinée au dépôt et à la diffusion de documents scientifiques de niveau recherche, publiés ou non, émanant des établissements d'enseignement et de recherche français ou étrangers, des laboratoires publics ou privés.

\section{(ㅇ)(1) $\$$}

Distributed under a Creative Commons Attribution - NonCommercial - NoDerivatives| 4.0 


\title{
De la philosophie morale humienne à l'éthique du care : un billet
}

\section{aller-retour}

\author{
Céline Bonicco-Donato
}

Unanimes dans leur critique de la morale kantienne dont elles dénoncent le caractère non seulement réducteur, incapable de rendre compte de l'ensemble des problèmes moraux $^{1}$, mais également méprisant, dans la mesure où les phénomènes qu'elle exclut sont jugés inférieurs à ceux dont elle traite, certaines théoriciennes de l'éthique du care n'hésitent pas à revendiquer leur filiation avec une autre tradition de la philosophie morale plus marginale, la théorie des sentiments moraux ${ }^{2}$, telle qu'elle s'élabore en Ecosse au XVIIIème siècle. Joan Tronto s'avère la plus précise sur ce point dans Un monde vulnérable. Soucieuse d'élaborer une véritable théorie du care en dépassant les impasses théoriques de Carol Gilligan qui, en comprenant l'éthique du souci des autres comme une morale proprement féminine, risque de conforter la dévaluation du deuxième sexe en maintenant une distinction entre la morale publique masculine et la morale privée féminine, elle s'emploie, en effet, à montrer que les sentiments moraux, affects et autres feelings, loin d'être l'apanage des femmes, ont été au cœur d'une théorie élaborée par des hommes. «Comme le suggère la lecture des penseurs écossais des Lumières, les hommes de ce siècle témoignaient de sentiments fréquemment attribués aux femmes : sensibilité morale, attachement aux autres et à la communauté. Ce fait historique affaiblit la portée de l'idée qu'une sorte de relation biologique, psychologique ou culturelle à validité universelle relierait les femmes aux sentiments moraux $»^{3}$. Attentive au champ notionnel déployé par Hutcheson, Smith et Hume, elle reconnait à ces trois philosophes le mérite d'aborder le champ moral à partir de la responsabilité et des relations intersubjectives concrètes, en lieu et place du droit et des règles formelles et abstraites. Antérieure à la morale kantienne, cette autre tradition offre donc un

\footnotetext{
${ }^{1}$ Voir notamment de manière explicite J Tronto, Un monde vulnérable, Paris, La Découverte, 2009, p. 56 sq. et de manière implicite Carol Gilligan, A Different Voice, Harvard, Harvard University Press, 2009 p. 74.

${ }^{2}$ Voir J. Tronto, Un monde vulnérable, op. cit., p. 55 sq.; A. Baier, «What do Women want in a Moral Theory », Noûs, Vol. 19, n 1, 1985, p. 57 et «Hume: The Woman's Moral Theorist? », in E. F. Kittay et D. T. Meyers (eds), Women and Moral Theory, U.S.A, Rowman \& Littlefield, 1987, p. 42 sq. ; ainsi que S. Haber, «Ethique du care et problématique féministe dans la discussion américaine actuelle », in S. Laugier et $\mathrm{P}$. Paperman (eds), Le souci des autres, Paris, éditions de l'EHESS, 2011, p. 188.

${ }^{3}$ J. Tronto, Un monde vulnérable, op. cit., p. 93.
} 
modèle alternatif pertinent, montrant la possibilité d'élaborer une théorie du care transcendant la question du genre, et offrant les pistes conceptuelles pour la réaliser.

Néanmoins, cette appréciation positive n'est pas dénuée d'ambiguïtés. En effet, si l'éthique du care possède « une forte ressemblance » avec le « modèle des sentiments moraux du XVIIIème siècle ${ }^{4}$, il ne saurait être question d'affirmer une stricte identité entre les deux ni même d'envisager un « retour aux idées des penseurs écossais des Lumières ${ }^{5}$, puisque les sentiments moraux qu'ils mettent au cœur de leur théorie suscitent des problèmes qu'ils ne résolvent qu'en faisant intervenir, de manière insidieuse, la raison. Selon elle, ils n'assumeraient donc pas complètement leur parti pris affectif et contextuel, exprimant, en ce sens, une crise de confiance dans les sentiments qui s'expliquerait, en partie, par la modification des conditions sociales au XVIIIème et l'accroissement de la distance entre les individus nous rendant moins sensibles les uns aux autres. Le recours à la théorie des sentiments moraux se fait donc pour Tronto sur un mode paradoxal. Elle lui accorde le mérite de souligner les problèmes que doit résoudre toute morale non universaliste mais lui reproche de le faire par des concepts extrinsèques à sa logique affective. Si Joan Tronto a incontestablement raison de marquer une différence entre l'éthique du care et la théorie des sentiments moraux, sa critique s'avère cependantdiscutable, nous semble-t-il, sur le fond. En effet, la pierre d'achoppement entre ces deux philosophies morales réside moins dans l'intervention ou dans l'éviction de la raison que dans la manière d'aborder la morale. La théorie des sentiments moraux est avant tout une philosophie du jugement moral, attentive à la manière dont nous attribuons le caractère bon ou mauvais à une action et vertueux ou vicieux à un agent, alors que l'éthique du care se donne principalement comme une philosophie de l'action morale. Est moins en cause l'évaluation que la pratique : «cette morale trouve sa meilleure expression (...) sous celle de (...) la 'mise en œuvre du care'» ${ }^{6}$.

Aussi nous proposons nous, dans cet article, de réévaluer la dette de l'éthique du care à l'égard de la théorie des sentiments moraux, en nous attachant à la version qu'en donne David Hume dans son Traité de la nature humaine et dans l'Enquête sur les principes de la morale, dans la mesure où elle est habituellement analysée en histoire de la philosophie comme l'alternative la plus cohérente à la morale kantienne.Il s'agit bien, pour nous, de rendre raison à l'appréciation de Tronto, en mesurant l'originalité de la conception humienne de la morale dans le champ philosophique, qui justifie pleinement l'importance qu'elle lui

\footnotetext{
${ }^{4}$ Ibid., p. 190.

5 ibid., p. 68.

${ }^{6}$ Ibid., p. 116.
} 
accorde, mais également de souligner que les divergences ne s'établissent pas là où elle le dit. A notre sens, il convient moins de pointer des insuffisances dans l'argumentation de Hume qu'une différence d'orientation. Cette mesure prise, il devient alors possible d'opérer une « reconstruction $»^{7}$ de la philosophie morale humienne à l'aune de l'éthique du care pour voir quel secours elle peut lui apporter pour résoudre deux de ses problèmes immanents : la question du localisme (si l'on a tendance à se soucier et à se préoccuper des personnes les plus proches de nous, l'éthique du care n'est-elle pas irrémédiablement entachée d'une partialité qui limite considérablement sa portée ?) et son articulation à la justice (est-il possible de penser le care non seulement comme un concept moral mais également comme un concept politique ?).

I. «Ce qui est actuellement appelé 'moralité des femmes' ressemble de manière frappante à la pensée morale de l'Ecosse des Lumières » 8

La référence à la théorie des sentiments moraux joue un rôle stratégique dans l'argumentation de Tronto : invalider la localisation de l'éthique du care dans une sphère exclusivement féminine, en montrant que des hommes ont pu conceptualiser une approche de la morale offrant un air de famille avec celle-ci. Dans cette perspective, elle attache une attention particulière à la philosophie humienne dans la mesure où le concept de sympathie qui joue un rôle majeur dans l'économie des livres II et III du Traité de la nature humaine permet de penser le souci des autres et la sollicitude qui sont au cœur de la perspective du care. De l'analyse de Tronto se dégagent quatre caractéristiques de la philosophie morale humienne qui peuvent guider l'élaboration de la théorie qu'elle veut accomplir, en présentant un paradigme alternatif à la morale universaliste kantienne.

De manière cohérente avec son projet, Tronto souligne tout d'abord le rôle que Hume fait jouer à la sympathie dans la sphère morale, qui résulte d'une invalidation de celui de la raison. Ce point lui apparaît tout à fait essentiel puisqu'elle reproche à l'approche dominante d'exiger que « la morale soit tirée de la raison humaine, sous la forme de principes

\footnotetext{
${ }^{7}$ Nous suivons ici la perspective qu'adopte Annette Baier à propos de la justice humienne dans « What do Women want in a Moral Theory», op. cit., p. 57. Elle avance l'idée qu'elle a forgé le concept de confiance auquel elle accorde une importance capitale à partir d'une « réélaboration» de son analyse dans le Traité de la nature humaine. Il s'agit donc de s'appuyer sur les textes du philosophe en les interrogeant à partir d'une préoccupation qui leur est extrinsèque, quoique non étrangère, pour relever ce qu'ils peuvent nous apprendre dans cette perspective.

${ }^{8}$ J. Tronto, Un monde vulnérable, op. cit., p. 49.
} 
universels, abstraits et formels $»^{9}$. Cela conduit, en effet, à considérer comme non morales les actions motivées par la sollicitude et à envisager l'agent moral comme un acteur impartial et désengagé, ce qui relève de la pure fiction ${ }^{10}$. Une telle conséquence apparaît de manière évidente dans la réponse de Lawrence Kohlberg à Carol Gilligan qui mettait en cause sa compréhension évolutionniste de la raison pratique kantienne et critiquait sa compréhension de l'acquisition de principes déontologiques rationnels commandant nos choix comme le stade le plus évolué du développement moral. Loin de reconnaître qu'il existerait une « voix différente » pour penser la manière dont nous agissons moralement, comme l'invite Gilligan, Kohlberg affirme que « le premier sens du mot morale correspond (...) au point de vue moral (qui) insiste sur les attributs d'impartialité, d'universalisation, l'effort et la volonté de parvenir à un accord ou à un consensus entre les êtres humains sur ce qui est bien $»^{11}$.

Or comme le note Tronto, c'est précisément contre une telle compréhension de la morale que s'est positionné David Hume dans le livre III de son Traité de la nature humaine. S'il ne pouvait réfuter la morale kantienne postérieure à la sienne, il n'en demeure pas moins vrai que les premières lignes de cet ouvrage sont consacrées à identifier la source des distinctions morales et que la réponse tombe sans appel dès le titre de la section I : «les distinctions morales ne proviennent pas de la raison $»^{12}$. Se positionnant contre les philosophes rationalistes de son temps, notamment Balguy et Clarke, il considère qu'il existe un « sens moral » selon le titre de la section II. Puisque ce dernier ne saurait s'identifier à une faculté dont Hume n'a jamais cessé de dénoncer le caractère fictionnel, il faut simplement entendre par là que nous distinguons le bien du mal au moyen d'un feeling d'une nature particulière. Ainsi considère-t-il que «le vice vous échappe totalement tant que vous considérez l'objet. Vous ne pourrez jamais le trouver avant d'orienter la réflexion vers votre propre cœur et de constater qu'un sentiment de désapprobation s'élève en vous contre cet acte. Voilà un fait, mais il est l'affaire de l'impression et pas de la raison ${ }^{13}$. Le critère pour juger une action vertueuse n'est donc pas sa conformité objective à un principe universel, établi rationnellement, mais la présence en nous d'un sentiment particulier. La sensation d'une peine ou d'un plaisir dont il faudra préciser la spécificité, puisque Hume insiste sur ce point, nous amène à attribuer une valeur morale à l'agent ou à l'action les ayant provoqués. Autrement dit, lesqualités approuvées par le sens moral, le vice et la vertu, ne doivent pas être

\footnotetext{
${ }^{9}$ Ibid., p. 57.

${ }^{10}$ Voir T. Nagel, The Moral Point of View. The Rational Basis of Ethics, Randon House, New York, 1965.

${ }^{11}$ L. Kohlberg, C. Levine et A Hewer, Moral Stages. A Current Formulation and a Response to Critics, New York, Karger, 1983, p. 229.

${ }^{12}$ D. Hume, Traité de la nature humaine, Paris, GF-Flammarion, 1993, III, 1, 1, p. 49.

${ }^{13}$ Ibid., III, 1, 1, 26, p. 64.
} 
comprises comme des qualités premières des objets, découvertes rationnellement, mais comme des qualités secondes relatives à l'observation sensible de l'objet.

Cette analyse minutieusement argumentée par Hume dans la première section du livre III du Traité implique une détermination essentielle de la sphère morale selon les théoriciennes du care : son caractère problématique et ambigu que le rationalisme moral tend à édulcorer. En effet, dans la mesure où, pour Hume, les sentiments n'ont pas de contenu représentatif $^{14}$, ils ne peuvent être dits vrais ou faux ${ }^{15}$, ce qui différencie de manière radicale la sphère morale de celle de la connaissance et interdit à celui qui évalue d'être jamais totalement sûr de son jugement et de pouvoir le démontrer. Le plaisir ou le déplaisir particulier éprouvé, nous poussant à considérer une action comme bonne ou mauvaise, échappent à la valeur de vérité. En ce sens, la théorie humienne vient donner une assise épistémologique à ce que les analyses sociologiques des différentes tâches relevant du care giving mettent en avant : les conflits incessants qui se posent aux acteurs. «Que faut-il faire quand on retrouve deux vieilles dames tous les matins dans le même lit? Faut-il tolérer qu'un patient boive en cachette ? Ce patient mourant préfère fumer plutôt que de s'alimenter. Va-ton lui accorder ce dernier plaisir ? Ou bien va-t-on céder à sa famille qui, refusant l'imminence du décès, exige des aides-soignantes qu'elles confisquent les cigarettes et le contraignent à manger ? ${ }^{16}$. Autant de dilemmes quant au choix de l'action pertinente à déployer mais qui se posent en premier lieu au niveau de l'évaluation de la situation.

Reste à comprendre en quoi consiste la particularité des feelings à l'origine de nos distinctions morales. «Ce n'est que lorsqu'un caractère est considéré en général, sans référence à notre intérêt particulier, qu'il produit une impression ou un sentiment qui le distinguent comme moralement bon ou mauvais $»^{17}$. Ainsi les sentiments moraux, plaisir ou déplaisir, ne peuvent être éprouvés qu'à condition d'adopter une position désintéressée à l'égard de ceux évalués. Ce caractère désintéressé ne signifie nullement un désengagement à l'instar de celui du sujet moral kantien mais simplement que nous nous intéressons aux actions de l'autre, dans la mesure où nous y sommes sensibles sans avoir un intérêt particulier, direct et égoïste à le faire. Or seule la sympathie semble à même de rendre compte de cette capacité.

Définie dans le livre II du Traité comme la qualité tout à fait remarquable permettant de « recevoir par communication (les) inclinations et (les) sentiments (d'autrui)

\footnotetext{
${ }^{14}$ Voir D. Hume, Traité de la nature humaine, Paris, GF-Flammarion, 1991, II, 3, 3, 3, p. 270.

${ }^{15}$ Ibid., III, 1, 1, 9, p. 52

${ }^{16} \mathrm{P}$. Molinier, « Le care à l'épreuve du travail », Le souci des autres, op. cit., p. 347.

${ }^{17}$ D. Hume, Traité de la nature humaine, op. cit., III, 1, 2, 4, p. 68.
} 
fussent-ils différents des nôtres, voire contraires aux nôtres ${ }^{18}$, elle se compose de deux opérations cognitives : l'inférence de l'idée de ce qu'éprouve l'autre et la conversion de cette idée en impression, de telle sorte que le sympathisant partage à la première personne les impressions du sympathisé. Si la sympathie joue un rôle central dans la communication des passions, elle permet également de comprendre l'approbation morale qui repose sur le fait d'éprouver un plaisir désintéressé, comme nous l'avons vu. C'est à ce titre que Hume la fait intervenir dans son analyse du livre III où il montre que les qualités jugées vertueuses, de manière générale, sont utiles ou agréables à la personne qui les possède ou à ceux qui sont concernées par son action, et procurent par là même du plaisir à leur possesseur ou à leurs bénéficiaires. Or par sympathie, je peux partager moi-même ce plaisir indépendamment de mon intérêt immédiat, en éprouvant les impressions d'autrui. Je me sentirai alors en mesure de juger moralement bonnes ces qualités et l'action qu'elles ont motivée. La même analyse est réitérable lorsque le déplaisir spécifique du vice est en jeu. Permettant de se soucier de ce qu'éprouvent les autres en l'endossant soi-même à la première personne, la sympathie parvient par là même à rendre compte de la nature spécifique des affectsservant de critère au jugement moral.

La deuxième caractéristique de l'éthique du care relevée par Gilligan dont Tronto trouve les prémisses dans la théorie des sentiments moraux, notamment chez Hume, réside dans l'accent mis sur les relations intersubjectives plutôt que sur les droits et les règles. Le domaine moral, selon elle, doit moins s'aborder à partir de principes universels abstraits définissant les droits et les devoirs réciproques des agents en présence qu'à partir de la nature de la relation qui nous unit aux autres. Ainsi le point de vue moral apparaît-il comme un point de vue irrémédiablement situé et engagé. Or cette particularité peut se comprendre en raison du caractère plus ou moins extensif de la sympathie qui nous invite à penser, selon Hume, différentes situations où notre jugement moral se voit altéré à des degrés variables selon la proximité que nous avons avec les personnes avec lesquelles nous sympathisons. En effet, si le jugement moral repose sur notre capacité à éprouver ce qu'éprouve l'autre pour éventuellement ressentir un feeling particulier de plaisir ou de déplaisir, la question de notre relation avec ce dernier et de la force de notre attachement est décisive. Dès le livre II du Traité de la nature humaine, Hume pointe du doigt le fait que nous sympathisons plus avec le proche qu'avec le lointain ${ }^{19}$, que nous partageons plus aisément les impressions des personnes

\footnotetext{
${ }^{18}$ Ibid., II, 1, 11, 2, p. 155-156.

${ }^{19}$ Voir ibid., II, 2, 4, 6, p. 201.
} 
qui nous ressemblent et dont nous sommes familiers, ce qui le conduit à aborder la question du jugement moral dans la dernière section du livre III non pas à partir de la recherche de principes présidant à son déploiement mais à partir de la force du lien qui nous unit à son objet. Le problème moral fondamental ne réside pas dans notre capacité à suivre des règles élaborées rationnellement mais dans celle à nous sentir potentiellement concernés par l'autre ou l'étranger.

Cette perspective engage une épistémologie et une manière d'élaborer la philosophie morale très originales. En effet, la théorie élaborée ne peut se donner comme une déduction logique de principes formels universels donnant la clé de l'évaluation et de l'action morales. Bien au contraire, elle doit emprunter le détour de la narration et du cas particulier pour réfléchir aux différentes manières dont se modalisent nos engagements à l'égard de ceux que nous évoluons. Ainsi les textes humiens abondent-ils de micro-fictions toutes plus savoureuses les unes que les autres depuis celle de l'homme qui se soucie davantage du bris de son miroir que de l'incendie d'une maison située à quelques lieux de là ${ }^{20}$ à celle de notre sollicitude pour l'homme qui tousse ${ }^{21}$ en passant par l'histoire de l'étranger qui porte un intérêt croissant aux habitants de la ville dans laquelle il s'établit ${ }^{22}$. La démarche morale à laquelle Hume invite son lecteur répond à bien des égards à la caractérisation du raisonnement du care donné par Patricia Paperman : il «ne valide pas ses réponses en référence à des principes généraux mais prend la forme d'une narration où les détails concrets, spécifiques des situations toujours particulières prennent sens et deviennent intelligibles dans les contextes de vie des personnes $»^{23}$.

Le troisième mérite que Tronto accorde à la philosophie morale humienne découle directement du précédent : loin d'envisager la morale comme un espace isomorphe et abstrait où les principes déduits s'appliqueraient de manière universelle dans toutes les situations, le philosophe écossais en appelle «à l'imagination morale, au caractère et aux actions de chacun » pour « répondre à la complexité de la situation donnée ${ }^{24}$. Dans chaque situation, il convient de mettre à l'épreuve la force de son engagement et de sa réceptivité à l'autre. L'impossibilité de vérifier la pertinence de son évaluation qui échappe, comme nous l'avons vu, à la valeur de vérité, renforce cette incertitude et invite à envisager chaque situation

\footnotetext{
${ }^{20}$ Ibid., III, 3, 1, 26, p. 212.

${ }^{21}$ D. Hume, Enquête sur les principes de la morale, Paris, GF-Flammarion, 1991, V, 2, p. 133.

${ }^{22}$ D. Hume, Traité de la nature humaine, op. cit., II, 3, 10, 12, p. 313.

${ }^{23}$ P. Paperman, Care et sentiments, Paris, P. U. F., 2013, p. 14.

${ }^{24}$ J. Tronto, «Au-delà d'une différence de genre », Le souci des autres, op. cit., p. 64.
} 
morale comme une situation singulière où se joue le conflit du particulier et du général, le souci du proche et l'appel à la sollicitude du lointain.

Assez curieusement, Tronto ne relève pas dans la philosophie humienne une dernière dimension qu'elle considère pourtant essentielle dans l'élaboration d'une théorie du care digne de ce nom : la critique de la conception cartésienne et kantienne du sujet comme entité autonome, indépendante et suffisante, qui conduit à poser l'interdépendance et la vulnérabilité comme des attributs subjectifs irrécusables. «Si nous n'avons pas tous besoin de l'aide des autres en toutes circonstances, notre autonomie ne s'acquiert qu'après une longue période de dépendance et, à bien des égards, nous restons dépendants des autres tout au long de notre vie : cela fait partie de la condition humaine $»^{25}$. Or Hume procède à une destruction massive de la fiction de l'identité personnelle dans le livre I du Traité2 ${ }^{26}$,en montrant qu'il n'existe aucune impression de soi qui justifie la vérité de l'idée que nous nous faisons de nous-mêmes. Le livre II présente l'identité personnelle comme une construction sociale s'élaborant dans les relations intersubjectives, grâce à la sympathie, pour autant que la prise en compte de la manière dont les gens se soucient de nous aide à se former une image de soi, un soi qui apparaît alors assuré et rassuré par autre que lui. L'identité personnelle chez Hume apparaît comme le fruit paradoxal d'une subjectivation s'effectuant moins dans un décentrement puisque cela supposerait l'existence d'un centre préalable, que dans une structure de relations externes : on ne devient soi qu'en s'associant et en communiquant avec les autres par le partage de valeurs communes. Deviennent dès lors compréhensibles les raisons de la focalisation humienne sur la nature de la relation entre le sujet du jugement moral et son objet dans une situation donnée, en lieu et place de l'intérêt pour l'application individuelle et abstraite des principes par un sujet désincarné.

II/ Quelles limites pour la philosophie morale humienne à l'aune de l'éthique du care?

Si Joan Tronto sait gré à David Hume d'avoir élaboré une philosophie morale proposant un paradigme sentimentaliste et contextualiste, elle pointe également du doigt deux limites intrinsèquement liées, signes d'un manque de confiance dans les sentiments moraux. Selon elle, Hume règlerait le problème du caractère plus ou moins extensif de la sympathie

\footnotetext{
${ }^{25} \mathrm{~J}$. Tronto, Un monde vulnérable, op. cit., p. 212

${ }^{26}$ Voir D. Hume, Traité de la nature humaine, Paris, GF-Flammarion, 1995, I, 4, 6, p. 342 sq.
} 
par l'intervention de la raison : évincée par la porte principale dans un premier temps comme incapable de distinguer les valeurs morales, la faculté rationnelle se réinviterait par l'issue de secours pour corriger le caractère partial de la sympathie. Puisque nous nous soucions plus du proche que du lointain, il nous faudrait amender cette tendance initiale à la partialité par une faculté stable, abstraite et universelle, ignorant les particularismes et les variations. L'incapacité de la sympathie à surmonter la distance qui peut exister entre deux individus permettrait de comprendre le deuxième manque que pointe Tronto : la distinction qui existe, dans le livre III du Traité, entre la sphère de la morale et la sphère de la justice. Si la morale repose sur des sentiments mais que ceux-ci ont du mal à se déployer entre des individus qui sont étrangers les uns aux autres, un système de régulation supérieur, reposant sur le droit, apparaît nécessaire. Ainsi Hume subordonnerait-il, au moins du point de vue de l'extension, la morale à la justice, puisque la première serait incapable de régler seules les relations humaines. Hume suggèrerait que «nous ne pouvons espérer que toutes les personnes agissent conformément au bien les unes des autres ou comprennent la nécessité de sacrifier leurs intérêts propres aux besoins des autres. Les sociétés humaines créent au contraire des systèmes de justice pour assurer un équilibre entre les activités qui nous concernent en propre et celle qui concernent les autres $»^{27}$. Tronto voit dans cette analyse la conséquence théorique d'un changement social : l'accroissement de la distance entre les individus au XVIIIème siècle en raison de la montée du libéralisme, qui rendrait prégnante la question du contrôle de relations intersubjectives devenues trop distendues, froides et complexes pour pouvoir être régulées par la simple sphère affective.

Mais si Joan Tronto a incontestablement raison de considérer que la philosophie morale humienne ne fait que donner les linéaments de l'éthique du care, il ne nous semble cependant pas que sa critique lui rende totalement justice. En effet, si Hume fait intervenir la raison pour corriger la partialité initiale de la sympathie, il ne faut pas oublier que celle-là n'est jamais qu'une « détermination calme et générale des passions, fondée sur quelque vue lointaine ou réflexion distante $»^{28}$, autrement dit la correction de la partialité de la sympathie n'est pas l'œuvre d'une faculté extérieure au processus mais se fait de manière immanente,par un progrès des sentiments ${ }^{29}$ pour reprendre le titre d'un ouvrage d'Annette Baier commentant l'œuvre humienne. Loin de faire intervenir une autre instance, Hume s'efforce de penser,depuis la sphère affectiveelle-même, la résolution du particularisme qui semble

\footnotetext{
${ }^{27}$ J. Tronto, Un monde vulnérable, op. cit., p. 78.

${ }^{28}$ D. Hume, Traité de la nature humaine, op. cit., III, 3, 1, 18, p. 205.

${ }^{29}$ Voir A. Baier, A Progress of Sentiments, Cambridge, Harvard University Press, 1991.
} 
condamner toute morale reposant sur une base non rationnelle à une portée très limitée cantonnée dans la sphère familiale et amicale. Ce point nous semble absolument décisif pour apporter des éléments de réponse à une question cruciale posée par Tronto sur laquelle nous reviendrons ultérieurement: "Comment garantir que les personnes engagées dans la distribution et la réception du soin soient capables de se dégager de leurs propres préoccupations locales et de s'intéresser à des besoins généraux de care $? »^{30}$. Le mérite de l'analyse humienne est de penser une autorégulation de la sympathie dépassant sa partialité initiale pour accéder à un souci général des autres qui n'aura peut-être pas la même chaleur affective que celui particulier du proche mais se révèlera suffisant pour nous permettre de nous intéresser les uns aux autres et agir en conséquence. Loin d'être la reconduction d'un irénique modèle familial où les relations morales devraient se penser comme le prolongement de celles unissant une mère à son enfant, l'éthique humienne prend acte de la distance qui sépare les individus pour penser à quelles conditions un processus affectif, «le jeu des sentiments $»^{31}$, peut la transcender, sur l'ordre du commesi, sans pouvoir jamais l'abolir.

La distinction que Tronto voit entre la sphère morale et la sphère de la justice dans la philosophie humienne semble également erronée. Et ce, pour au moins trois raisons. D’une part, la justice apparaît bien comme une vertu artificielle mais les conventions qui la renforcent ont été forgées à partir de la sympathie. En ce sens, il ne faut pas opposer comme le fait Tronto l'idée naturelle de bienveillance sur laquelle reposerait la justice et les conventions humaines qui la consolideraient ${ }^{32}$, il faut plutôt penser comment la capacité naturelle à ressentir ce que ressentent les autres peut engendrer des institutions. Est moins à l'origine de la justice la bienveillance que la sympathie limitée aux proches: notre sens de l'intérêt personnel et de l'intérêt pour notre clan nous pousse à passer des conventions avec les autres hommes qui effectuent le même raisonnement que nous. Pour mieux se satisfaire qu'elle ne peut le faire spontanément, notre partialité initiale choisit de se brider en inventant des artifices. Bien qu'artificielle, la justice ne peut donc être dite arbitraire pour autant qu'elle procède de principes naturels se limitant selon une logique, elle aussi, naturelle. L'artifice humien se présente comme une seconde nature. Loin d'être des adjuvants extrinsèques à la sympathie, permettant de réguler les relations entre personnes distances, les conventions de justice sont produites par la sympathie et peuvent, à ce titre, être définies comme les moyens qu'elle invente pour corriger sa partialité initiale.

\footnotetext{
${ }^{30}$ J. Tronto, Un monde vulnérable, op. cit., p. 190.

${ }^{31}$ D. Hume, Enquête sur les principes de la morale, op. cit., V, 2, p. 139.

${ }^{32}$ Voir J. Tronto, Un monde vulnérable, op. cit., p. 78.
} 
D'autre part, il existe des «rudiments de justice ${ }^{33}$ dans la famille. Hume n'oppose nullement la morale inhérente à la sphère privée familiale à la justice immanente à la sphère publique. Il pense au contraire que les conventions sociales ne font qu'instituer ce qui est vécu de manière spontanée dans le cercle des proches. Si l'amplification passe nécessairement par un refroidissement de l'engagement, il n'en demeure pas moins vrai que Hume décrit une histoire naturelle de l'institution de justice depuis la sphère familiale, en lui assignant par là même comme tâche de réaliser dans la société le sens de l'intérêt commun, vécu immédiatement par les personnes proches les unes des autres. La justice humienne possède donc comme référence privilégiée les dispositions et les activités liées à l'attention et à la sollicitude.

Enfin, la justice fait l'objet dans la philosophie humienne d'une évaluation morale.Autrement dit, un ensemble de conventions artificielles inventées dans un premier temps pour permettre aux hommes de vivre en paix les uns avec les autres, répondant donc à un intérêt sinon égoïste du moins partial (il s'agit pour les hommes de protéger leurs familles et leurs proches) finit par être jugé comme bon pour autant qu'il possède une utilité publique. Les hommes cessent donc de lui reconnaître un intérêt particulier pour faire valoir son intérêt général, lui permettant d'être associé,à ce titre, à l'idée de vertu. Ainsi la justice et la morale peuvent-elles coïncider sur ce point très précis : lorsqu'un système de conventions régule les relations humaines de manière non seulement utile pour les individus en particulier, mais utile pour la société en général, il est jugé bon. Si l'on se souvient que la politique, pour Hume, n'a d'autre utilité que de rendre plus efficace la justice en inventant des sanctions, l'on saisit toute l'importance de cette analyse pour penser la politisation du care que Joan Tronto appelle de ses vœux.

Est-ce à dire que toute l'éthique du care soit contenue dans la philosophie morale humienne ? Nullement ! Si la critique de Tronto peut être contestée dans son argumentation, elle ne doit pas l'être dans son intention. En effet, eu égard aux exigences d'une théorie du care, la philosophie humienne apparaît insuffisante, en raison du point de vue où elle se situe. Alors qu'elle se focalise sur le problème de l'évaluation morale, le care se définit, pour sa part, comme « une activité générique qui comprend tout ce que nous faisons pour maintenir, perpétuer et réparer notre 'monde', de sorte que nous puissions y vivre aussi bien que possible $»^{34}$. Autrement dit, Hume envisage essentiellement le problème moral sous l'angle du

\footnotetext{
${ }^{33}$ D. Hume, Traité de la nature humaine, op. cit., III, 2, 2, 14, p. 93.

${ }^{34}$ B. Fisher et J. Tronto, « Toward a feminist theory of care », in E. Abel et M. Nelson (dir.), Circles of Care : Work and Identity in Women's Lives, Albany, State University of New York Press, 1991, p. 40.
} 
jugement (à quelles conditions, jugeons-nous une action bonne ou mauvaise, un caractère vertueux ou vicieux ?), la théorie du care essentiellement sous l'angle de la pratique (à quelles conditions, accomplissons-nous une bonne action ?).

La philosophie morale humienne apparaît avant tout comme une théorie descriptive mettant au jour les principes de la nature humaine permettant de comprendre le fonctionnement du jugement moral, alors que l'éthique du care n'exclut pas une visée normative, du moins une perspective perfectionniste. Si Hume rencontre le problème de l'action morale dans un second temps, puisque l'homme qui juge une action bonne peut se sentir appelé à l'accomplir ${ }^{35}$, il n'en demeure pas moins vrai que son angle d'approche de la sphère éthique est beaucoup plus contemplatif que celui des théoriciennes du care. Que la sympathie nous permette de nous soucier des autres se manifeste moins sous la forme de la sollicitude directe que sous celle d'un intérêt pour la valeur de leurs actions qui ne se traduit pas nécessairement par une action engagée. «Ma sympathie pour un autre homme peut me faire éprouver, quand se présente un objet qui a tendance à lui donner du désagrément, le sentiment de la douleur et de la désapprobation, bien que je puisse ne pas vouloir sacrifier quoi que ce soit de mon intérêt personnel, ou contrarier l'une de mes passions pour satisfaire cet homme $»^{36}$. A l'inverse, Joan Tronto ne cesse de mettre en garde contre la compréhension exclusive du care en termes de disposition pour inviter à le penser aussi comme pratique ${ }^{37}$, ce qui conduit à poser comme figure privilégiée de la morale, non pas le bon juge capable de s'intéresser aux actions des autres indépendamment de son propre intérêt pour évaluer le plaisir ou le déplaisir qu'elles procurent, mais le bon agent qui est non seulement capable de se soucier des autres mais qui «s'efforce de répondre aux demandes de soin auxquelles il est confronté dans sa vie $»^{38}$. Devient alors aisément compréhensible le caractère non seulement plus large mais aussi plus précis du care. D’une part, le care déborde la sphère des liens intersubjectifs puisque les demandes de soin peuvent être formulées par des individus mais aussi par «notre environnement ${ }^{39}$, si bien que la relation dyadique ne doit pas être pensée comme modèle absolu. D'autre part, s'il se conçoit d'abord sous l'angle d'un processus actif,

\footnotetext{
${ }^{35}$ Annette Baier dégage principalement à partir de l'Enquête sur les principes de la morale les traits constitutifs de la morale humienne envisagée du point de vue de la conduite. Jugeant vertueux l'homme qui procure du plaisir aux autres et à soi-même, on apprend à son tour à cultiver les traits de caractères susceptibles de procurer un tel plaisir pour devenir une «personne de bonne compagnie». Voir A. Baier, Moral prejudices. Essays on Ethics, Cambridge, Harvard University Press, 1994, p. 55 sq. Pour le caractère motivant des distinctions morales, voir D. Hume, Traité de la nature humaine, op. cit., III, 1, 1, 4-8, p. 50 sq.

${ }^{36}$ D. Hume, Traité de la nature humaine, op. cit., III, 3, 1, 23, p. 208-209.

${ }^{37}$ Voir J. Tronto, Un monde vulnérable, op. cit., p. 163.

${ }^{38}$ Ibid., p. 172.

${ }^{3939}$ Voir la définition de Fisher et Tronto in « Toward a feminist theory of care », in E. Abel et M. Nelson (dir.), Circles of Care: Work and Identity in Women's Lives, op. cit., p. 40.
} 
il comprend différentes phases qui dépassent la simple question du jugement pour marquer différents degrés d'engagement dans les situations : se soucier de, prendre en charge, prendre soin et recevoir le $\operatorname{soin}^{40}$, là où Hume distinguait simplement deux opérations cognitives (l'inférence de l'idée de ce qu'éprouve l'autre et la conversion de cette idée en impression) ainsi que des degrés d'extension.

Dans cette perspective devient aisément compréhensible la dernière lacune de la philosophie morale humienne à l'aune d'une théorie du care: l'absence de perspective critique. Soucieux de rendre compte d'un phénomène anthropologique, le jugement moral, à partir des principes de la nature humaine présidant à son déploiement, à une époque où le rationalisme ne domine pas de manière hégémonique, Hume ne peut se donner une tâche qui serait anachronique au XVIIIème siècle : mettre en question les hiérarchies de classe et de genre, en montrant que les activités du care sont le plus souvent exercées par des catégories défavorisées de la population et exclues de ce fait de la sphère théorique, le dénigrement conceptuel devenant l'expression, dans la sphère intellectuelle, de la dévalorisation de certains groupes sociaux. Mais si cette absence témoigne moins d'un manque que d'une orientation théorique différente s'expliquant en partie par les formes de vie du siècle des Lumières, il devient alors possible de s'appuyer sur son analyse, comme le fait Gilligan, pour mettre en cause une lecture idéologique des sentiments moraux justifiant leur cantonnement dans la sphère privée et familiale, au nom d'une prétendue nature féminine dont la construction sociale est devenue invisible.

III/ Que peut apporter la philosophie morale humienne à l'éthique du care?

Cette clarification des relations entre la sympathie humienne et le care contemporain permet de prendre la mesure exacte du secours qu'elle peut lui apporter. Si la théorie du care ne peut se contenter de répéter la philosophie des sentiments moraux, ce qui l'empêcherait de penser les réponses concrètes à apporter aux besoins des autres à partir de leur inscription dans des situations particulières où les conflits de classe, de race ou de genre sont omniprésents, il n'en demeure pas moins vrai qu'elle peut lui offrir certaines ressources conceptuelles, une fois assumée la différence de point de vue. En effet, en vertu des points de convergence précédemment soulignés, il devient possible de «reconstruire » certains développements du livre III du Traité de la nature humaine, dans la perspective de l'action et plus simplement du jugement moral, pour trouver des éléments de réponse aux deux

\footnotetext{
${ }^{40}$ J. Tronto, Un monde vulnérable, op. cit., p. 147 sq.
} 
difficultés soulignées par Tronto qui se posent à toute morale non universaliste. Loin de contourner ces difficultés, Hume y répond dans le cadre affectif et contextualiste qui est le sien, de sorte que son analyse peut les éclairer dans celui de l'éthique du care.

La première difficulté de l'éthique du care, que ne se prive pas d'exprimer ses adversaires, réside dans sa portée en apparence limitée, ce que Tronto nomme le localisme ${ }^{41}$ : «si les mères s'occupent de leurs propres enfants, pourquoi ne prendraient-elles pas davantage au sérieux les besoins de ceux-ci plutôt que ceux des enfants qui leur sont distants ? Pourquoi les Américains devaient-ils s'inquiéter de la distribution de nourriture au niveau mondial $? \gg^{42}$. Pour répondre à cette difficulté immanente à toute éthique se privant du secours de la raison qui permet de formuler des impératifs impartiaux, indépendants de nos relations avec les personnes concernées, les théoriciennes du care déploient deux stratégies. D'une part, montrer que les actions de sollicitude envers les proches qui composent notre expérience ordinaire possèdent une valeur morale ${ }^{43}$, donc que l'impartialité n'est pas une condition nécessaire del'action bonne, d'autre part que la notion de proximité perd de sa pertinence lorsque l'on prend la mesure de l'extension des activités relevant du care qui excèdent largement la sphère familiale : activités de soin à l'hôpital ou dans les maisons de retraite, aide à la personne dans son domicile, activités liées à la petite enfance, etc. Comme l'écrit Patricia Paperman, la notion de proximité «ne suffit pas à rendre compte de la complexité du processus ni surtout de son organisation sociale et politique $»^{44}$. Le recours à la philosophie humienne permet de donner plus de force à ces réponses en précisant leur argumentation.

En effet, dans le livre III du Traité de la nature humaine, Hume montre que le caractère initialement limité de la sympathie qui nous pousse à ressentir plus facilement ce que ressentent nos proches ne nous empêche pas de former des jugements moraux fermes et généraux puisque notre partialité initiale est susceptible d'être amendée. Là où son analyse s'avère particulièrement intéressante, dans le cadre d'une réflexion sur les exigences qu'une théorie du care doit satisfaire pour être conséquente, c'est que la correction envisagée ne nous voit pas devenir impartiaux mais ce que l'on pourrait appeler pluripartiaux. En effet, étendre sa sympathie pour Hume ne signifie pas devenir indifférent aux gens jugés, mais au contraire, apprendre à se sentir concernés par ce que leurs actions engendrent, donc intégrer dans sa

\footnotetext{
${ }^{41}$ Ibid., p. 190.

42 Ibid.

${ }^{43}$ Voir S. Wolf, « Morality and Partiality », Philosophical Perspectives, Vol. 6, Ethics, 1992, p. 243-259.

${ }^{44}$ P. Paperman, «D'une voix discordante. Désentimentaliser le care. Démoraliser l'éthique », in S. Laugier, P. Molinier, P. Paperman (eds), Qu'est-ce que le care ?, Paris, Payot, 2009, p. 100.
} 
partialité l'intérêt d'autrui, que ce soit celui que l'agent prend à ses propres actions ou celui des bénéficiaires extérieurs, source d'un plaisir pour ces deux catégories, que j'apprends moimême à éprouver comme évaluateur. «Le seul point de vue en lequel nos sentiments coïncident avec ceux des autres, c'est lorsque nous considérons la tendance d'une passion à être avantageuse ou dommageable pour ceux qui ont un rapport direct ou une relation immédiate avec celui qui la possède ${ }^{45}$. Il ne s'agit donc pas de mettre entre parenthèses son intérêt mais d'apprendre à s'intéresser à l'intérêt des autres : «bien que cet avantage ou ce dommage soient souvent très éloignés de nous, parfois, ils sont pourtant très proches et nous intéressent fortement par sympathie ${ }^{46}$. Le point de vue général nécessaire à la formulation d'un jugement authentiquement moral chez Hume se construit donc par inclusion de l'intérêt d'autrui dans son intérêt. Etre désintéressé signifie alors ni plus ni moins qu'être concerné par les intérêts d'autrui, quel que soit cet autre, c'est-à-dire indépendamment de nos relations avec lui.

$\mathrm{Si}$, fidèle à la perspective empiriste revendiquée dans l'introduction du Traité, l'analyse humienne se donne essentiellement comme une description, l'histoire naturelle de l'extension de la sympathie ne s'avère cependant pas dénuée d'enseignement pour réfléchir aux différents processus susceptibles d' "étendre notre domaine moral ${ }^{47}$, selon la belle expression de Tronto. En effet, dans la perspective humienne, la pluripartialité s'apprend dans la conversation avec les autres. La compagnie des autres hommes nous pousse à inclure leurs intérêts dans le nôtre pour pouvoir continuer à parler avec eux en comprenant le sens de leurs attachements $^{48}$. Quelles leçons en tirer dans le cadre de l'éthique du care ? Qu'apporter des réponses concrètes aux besoins d'autrui n'est pas inné mais se présente comme un processus complexe, s'améliorant par une éducation morale qui se joue tout au long de la vie. Il ne s'agit pas d'apprendre des préceptes abstraits mais de saisir que les différentes situations que nous traversons sont des configurations morales particulières donnant à saisir les intérêts des protagonistes autres que nous. Ainsi le localisme doit-il être moins aboli que déplacé, de manière à rendre proche de nous ce qui ne l'est pas, en s'efforçant d'y prêter attention. En ce sens, le rôle de l'éducation, de la littérature et des fictions en général nous confrontant à une

\footnotetext{
${ }^{45}$ D. Hume, Traité de la nature humaine, op. cit., III, 3, 3, 2, p. 229.

${ }^{46}$ Ibid.

${ }^{47}$ J. Tronto, Un monde vulnérable, op. cit., p. 195.

${ }^{48}$ Voir D. Hume, Traité de la nature humaine, op. cit., III, 3, 1, 15, p. 301 sq.
} 
multiplicité de scènes différentes ${ }^{49}$, de la rupture de l'entre-soi, et donc de la politique prennent tout leur sens.

Ce dernier point nous amène à poser le deuxième problème immanent à la théorie du care auquel la philosophie morale humienne peut apporter certains éléments de réponse, à condition de la lire dans cette perspective : son articulation avec une théorie de la justice. En effet, Tronto considère que séparer la morale de la justice, comme le fait Gilligan dans Une voix différente même lorsqu'elle envisage leur coopération possible, ne fait que conforter les frontières déjà établies entre privé et public, féminin et masculin, sentiments particuliers et règles universelles. A rebours, elle invite à inclure l'idée du care dans la conception de la justice pour que celle-ci soit vraiment complète (répondre aux besoins de care n'est pas seulement bon mais aussi juste), ce qui conduit à reconfigurer cette dernière pour qu'elle cesse de s'identifier, selon une logique libérale, à un traitement neutre des personnes et à une répartition impartiale des biens et avantages sociaux. La justice consiste également, selon elle, à offrir les conditions sociales et matérielles pour que les activités liées au care puissent s'exercer de la manière la plus satisfaisante pour ceux qui les prodiguent et ceux qui les déploient. Puisque ces pratiques concernent les activités dites de service qu'il s'agisse de service public ou de service à la personne, on mesure toute la portée de l'argument de Tronto : le care peut devenir une valeur démocratique, lorsqu'une société prend la mesure des besoins des différentes personnes et de leur interdépendance pour s'efforcer d'y répondre. C'est dans cette perspective que Tronto invite à transformer nos institutions, contribuant ainsi à politiser une théorie d'abord élaborée dans le champ moral. «L'introduction dans l'ordre politique des questions relatives à l'adéquation du care révèlera rapidement à quel point les organismes sociaux, les entreprises et les autres organisations bureaucratiques existantes servent peu les intérêts de leurs clients et des citoyens ordinaires. En conséquence, ceux-ci seront incités à exiger, par exemple, que les destinataires des soins soient intégrés à l'administration des services sociaux, ainsi que d'autres réformes démocratiques ${ }^{50}$. Il s'agit donc non seulement d'appréhender la sphère familiale comme une sphère politique où les pratiques genrées du care renvoient à des formes de hiérarchie mais également de penser que dans les pratiques non familiales du care, à l'hôpital, en maison de retraite, dans les crèches, aux guichets de la sécurité sociale, etc., se jouent des enjeux de pouvoir dans la répartition sociale des rôles et des tâches, dans l'image qui leur est attachée et dans les conditions de travail.

\footnotetext{
${ }^{49}$ Nous renvoyons sur ce point aux analyses de S. Laugier (dir.), Ethique, littérature, vie humaine, Paris, PUF., 2006.

${ }^{50}$ J. Tronto, Un monde vulnérable, op. cit., p. 228.
} 
Or la philosophie morale humienne semble à même de fournir des éléments intéressants pour penser cette reconfiguration de la justice en s'éloignant de sa conception dominante comme système de règles impartiales. Le livre III du Traité de la nature humaine la présente comme un artifice prolongeant et amplifiant la sympathie s'exerçant entre les proches. Ainsi, si l'on lit sa théorie dans la perspective qui nous intéresse, elle ne nous invite pas à penser l'inclusion du care dans la justice, selon la perspective de Tronto, mais l'inverse, sans donner pour autant une version maternaliste de cette dernière. Les institutions permettent de rendre effectif le sens de l'intérêt commun, non seulement dans la famille où, sans elles, il ne parviendrait pas à s'exprimer, mais également dans la société, en créant les médiations pour un «élargissement progressif de la confiance » ${ }^{51}$ selon l'expression d'Annette Baier. Que cette confiance ne soit ni spontanée ni aussi intense que dans les relations entre les proches n'exclut pas son importance. En ce sens, Hume n'établit aucune frontière étanche entre privé et public et pense la justice comme un produit de la sympathie, tout en en la présentant comme l'institution nécessaire pour que celle-ci puisse s'exercer au mieux. Si l'on suit cette perspective, l'inclusion de la justice dans le care n'exprime pas une subordination mais l'inverse : le care ne peut se déployer pleinement sans informer les relations sociales. Bref,il ne peut être moral sans être politique. Hume conçoit l'invention du gouvernement comme un adjuvant supplémentaire pour permettre à cette confiance de s'établir le plus sûrement possible, puisqu'il le pense comme un organe de régulation supplémentaire par l'administration de la sanction ${ }^{52}$. Si la conception humienne de la politique, essentiellement instrumentale, peut sembler restrictive, elle possède du moins le mérite de nous rappeler que l'organisation de la société n'est pas moralement neutre puisque, selon les institutions, différentes formes de vie peuvent ou non se déployer.

Loin d'être unilatérales, les relations entre la philosophie morale humienne et l'éthique du care se donnent sous la forme d'un chassé-croisé. Si les théoriciennes de l'éthique du care peuvent se revendiquer à juste titre héritières des différentes théories des sentiments moraux élaborées au XVIIIème siècle, la filiation n'est pas dénuée de paliers et de ruptures. En effet, le primat donné aux affects sur la raison, l'attention à la variabilité des relations et des situations, la mise en évidence de l'interdépendance n'empêchent pas une différence majeure de perspective sur le domaine moral, que la philosophie humienne

\footnotetext{
${ }^{51}$ A. Baier, « «What do Women want in a Moral Theory », op. cit., p. 57.

${ }^{52}$ D. Hume, Traité de la nature humaine, op. cit., III, 2, 7, 6, p. 146-147.
} 
exemplifie sans ambiguiité. L'éthique du care se donne comme une philosophie de l'action morale, attentive aux manières concrètes de répondre aux besoins des autres, alors que la théorie humienne se présente, avant tout, comme une élucidation du jugement moral, reléguant la question de l'engagement au second plan. Néanmoins, entre la sympathie humienne et le care contemporain, il n'existe pas simplement un aller simple, fût-il marqué par des seuils d'infléchissement. Il est, en effet, possible de relire les développements humiens, à l'aune de l'éthique du care, pour voir quels secours ils peuvent apporter à certaines de ses difficultés immanentes. Si les problèmes pointés du doigt par Tronto, propres à toute morale contextualiste, sont résolus par Hume sans réintroduction de la faculté rationnelle ni opposition des sentiments particuliers à des impératifs universels, contrairement à son diagnostic, il devient alors possible de s'appuyer sur l'analyse du philosophe pour dépasser le localisme et politiser le care. Il s'agit donc d'opérer un retour vers la philosophie humienne depuis l'éthique du souci des autres, pour saisir la portée de la sympathie éclairée par les exigences du care qui l'excèdent : donner à penser le dépassement de la partialité par une pluripartialité qui permet d'éviter le recours à une impartialité impensable dans le cadre d'une morale où les affects sont partie prenante et reconfigurer le concept de justice comme l'ensemble des institutions permettant le déploiement de pratiques dont le contenu possède une valeur morale. La sympathie humienne, principe à la fois moral et politique, nous rappelle que le care ne peut se distribuer sans le secours de l'artifice. 